\title{
A manufacturable smart dressing with oxygen delivery and sensing capability for chronic wound management
}

M. Ochoa, R. Rahimi, J. Zhou, H. Jiang, C. K. Yoon, et al.

M. Ochoa, R. Rahimi, J. Zhou, H. Jiang, C. K. Yoon, M. Oscai, V. Jain, T. Morken, R. H. Oliveira, D. Maddipatla, B. B. Narakathu, G. L. Campana, M. A. Zieger, R. Sood, M. Z. Atashbar, B. Ziaie, "A manufacturable smart dressing with oxygen delivery and sensing capability for chronic wound management," Proc. SPIE 10639, Micro- and Nanotechnology Sensors, Systems, and Applications X, 106391C (8 May 2018); doi: $10.1117 / 12.2306083$

SPIE. Event: SPIE Defense + Security, 2018, Orlando, Florida, United States 


\title{
A manufacturable smart dressing with oxygen delivery and sensing capability for chronic wound management
}

\author{
M. Ochoa ${ }^{a}$, R. Rahimi ${ }^{a}$, J. Zhou ${ }^{a}$, H. Jiang ${ }^{a}$, C. K. Yoon ${ }^{a}$, M. Oscai ${ }^{a}$, V. Jain ${ }^{a}$, T. Morken ${ }^{a}$, R. H. \\ Oliveira $^{\mathrm{a}}$, D. Maddipatla ${ }^{\mathrm{b}}$, B. B. Narakathu ${ }^{\mathrm{b}}$, G. L. Campana ${ }^{\mathrm{c}}$, M. A. Zieger ${ }^{\mathrm{c}}$, R. Sood ${ }^{\mathrm{c}}$, \\ M. Z. Atashbar ${ }^{\mathrm{b}}$, B. Ziaie ${ }^{* a}$ \\ ${ }^{a}$ Birck Nanotechnology Center. School of Electrical and Computer Engineering. Purdue University. \\ 1205 W State Street, West Lafayette, IN, USA 47907; \\ ${ }^{\mathrm{b}}$ Electrical and Computer Engineering Department, Western Michigan University, 4601 Campus \\ Drive B-236, Kalamazoo, MI, USA 49008. \\ 'Indiana University School of Medicine. 980 West Walnut Street, Building R3 Room C634, \\ Indianapolis, IN, USA, 48202.
}

\begin{abstract}
Chronic non-healing wounds, impact over 6.5 million Americans, costs in excess of \$25 billion to treat on an annual basis and its incidence is predicted to rise due to the prevalence of obesity and type- 2 diabetes. One of the primary complications often associated with chronic wounds is the improper functionality of the peripheral vasculature to deliver $\mathrm{O}_{2}$-rich blood to the tissue which leads to wound hypoxia. Although hyperbaric oxygen therapy are widely used and accepted as an effective approach to bolster tissue $\mathrm{O}_{2}$ levels in hypoxic chronic wounds, most of such treatments require bulky equipment and often expose large areas of the body to unnecessarily elevated oxygen concentrations that can damage healthy tissue. In this paper, we present a smart low-cost wound dressing with integrated oxygen sensor and delivery for locally generating and delivering oxygen to selected hypoxic regions on the wound. The dressing is fabricated on a biocompatible water resistant/hydrophobic paper-based substrate with printed optical oxygen sensors and patterned catalytic oxygen generating regions that are connected to a flexible microfluidic systems. Oxygen generation occurs by flowing $\mathrm{H}_{2} \mathrm{O}_{2}$ through the channels and chemical decomposition at the catalyst printed regions on the paper substrate. The hydrophobic paper provides structural stability and flexibility while simultaneously offering printability, selective gaseous filtering, and physical/chemical protection. The fabrication process take advantage of scalable manufacturing technologies including laser processing, inkjet printing, and lamination.
\end{abstract}

Keywords: Chronic wound, oxygen generation, oxygen sensor, smart dressing, flexible bioelectronics.

\section{INTRODUCTION}

Cutaneous wounds are a major health and financial burden for millions of people in the world. Chronic wounds, in particular, (e.g., diabetic foot and bed sores) impact over 6.5 million Americans per year and cost more than $\$ 25$ billion annually. Due to increasing obesity and diabetes compounded by an aging population, the occurrence of such wounds is on the rise ${ }^{1,2}$. Chronic wounds do not follow the standard cascade of biological processes (i.e., inflammation, proliferation, and maturation) by which common acute wounds heal ${ }^{3}$. Instead, their healing is impaired by conditions such as local hypoxia, irregular vascular structure, external mechanical pressure, and bacterial infections ${ }^{3-7}$. Current treatments are expensive and labor-intensive, relying on regular cleaning, debridement, oxygen therapy, surgery, and topical or systemic administration of antibiotics ${ }^{8-10}$.

A major component of treatment involves regular replacement of wound dressings (some of which contain therapeutic/antibacterial agents). Despite the many advances in wound care technologies, wound management and the selection of proper dressing that are adequate for each wound healing stage still rely heavily on visual inspection by the

* Corresponding author: Professor Babak Ziaie (bziaie@purdue.edu)

Micro- and Nanotechnology Sensors, Systems, and Applications X, edited by Thomas George,

Achyut K. Dutta, M. Saif Islam, Proc. of SPIE Vol. 10639, 106391C · C) 2018 SPIE

CCC code: $0277-786 \mathrm{X} / 18 / \$ 18 \cdot$ doi: $10.1117 / 12.2306083$ 
healthcare practitioner. Unfortunately, such subjective assessments cannot always provide precise insight into the status of the wound. As a result, researchers have been recently investigating the use of multi-parameter sensing such as $\mathrm{pH}$, oxygen, and infection detection to better diagnose wounds and aid in selecting appropriate treatments for optimal efficacy. Despite the many attempts in developing sensors and imaging devices for such applications, most of them up to now suffer from various limitations including the inability to operate in situ, lack of mechanical flexibility, use of cytotoxic materials that makes the device unusable in vivo, high cost of manufacturing, and a need for large external equipment (for stimulation or readout). In addition, most of these devices are implemented as simply improved feedback tools for medical specialists rather than integrating with therapeutic delivery systems for real-time automatic adjustment of therapy. An ideal dressing integrates sensors $(\mathrm{pH}$, oxygen, and inflammatory mediators), drug/cell delivery (antibiotics, growth factors, stem cells, and oxygen), and electronic intelligence to drastically improve wound care by measuring individual responses and enabling appropriate adjustments to therapy (i.e., precision $\backslash$ slash personalized wound care) ${ }^{11,12}$ via a closed-loop system, Figure 1 .

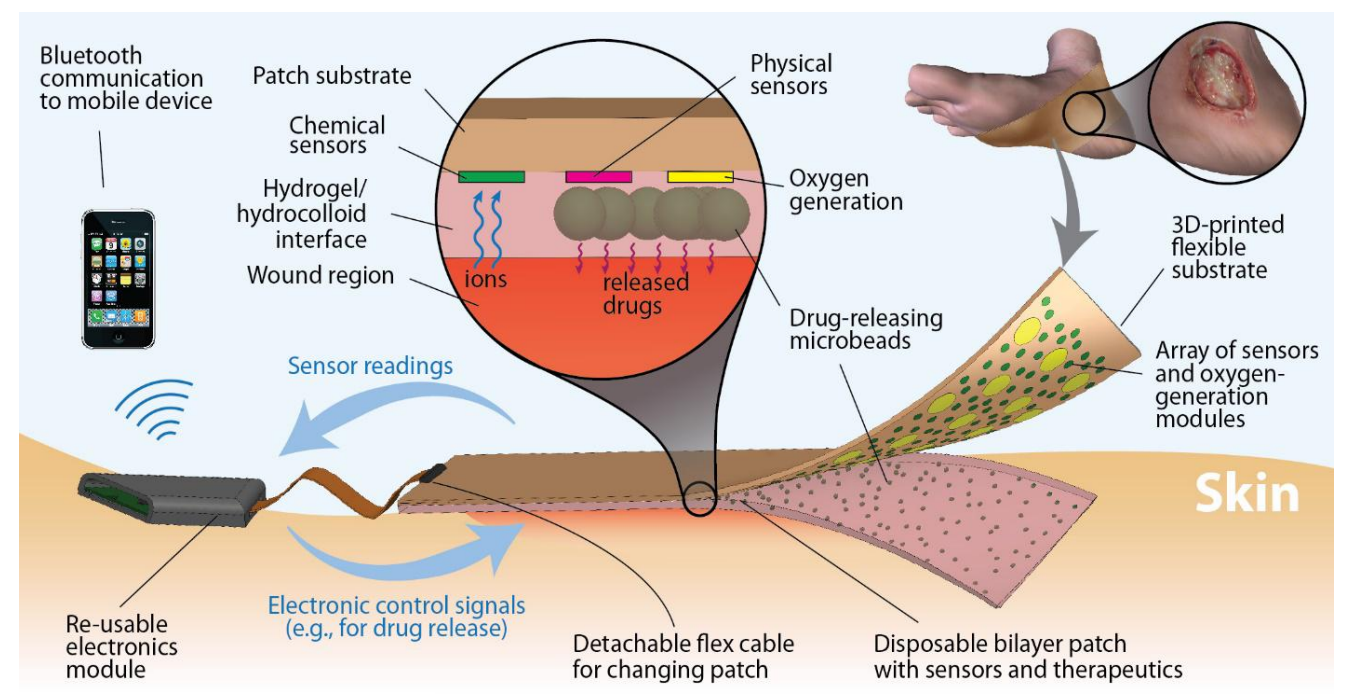

Figure 1: Vision of an integrated, closed-loop, smart wound dressing.

In order to develop a smart, multi-parameter wound healing system, it is important to address each of the many healing parameters, one at a time. Among the many issues hampering chronic wound healing, suboptimal oxygenation of the wound bed is one of the most critical and treatable. Whereas acute injuries often exhibit a sufficiently functional vascular network that provides adequate oxygen, chronic wounds lack such organization in the vascular network and are, thus, unable to receive sufficient oxygen to promote healing ${ }^{6,13,14}$. Although it is known that certain levels of hypoxia may trigger vascular regeneration, the severity and depth of chronic wounds can prevent adequate regeneration, causing wound ischemia. Today, hypoxia in chronic wounds is typically treated via hyperbaric oxygen chambers, which requires bulky equipment and often exposes large areas of the body to unnecessarily elevated oxygen concentrations that can damage healthy tissue ${ }^{15-17}$. Another, more practical, approach is topical oxygen therapy (TOT) in which atmospheric pressure oxygen is provided only at the wound site (e.g., by generating it or pumping it into a dressing), thus reducing the risk of hyperoxia while making treatment more comfortable for the patients. Various commercial systems exist which provide or generate oxygen to be delivered topically, Figure 2; however, they suffer from one or more of the following shortcomings: high cost (\$2000 per two weeks), inability to selectively deliver oxygen to specific wound regions (to address the hypoxic heterogeneity often found in chronic wounds), and a lack of modularity for incorporation with other wound sensing components.

As a first step towards the development of a multi-functional smart wound healing bandage, we developed a low-cost alternative for continuous $\mathrm{O}_{2}$ delivery comprising of an inexpensive, paper-based, biocompatible, flexible platform for locally generating and delivering oxygen to selected hypoxic regions. The platform takes advantage of recent developments in the fabrication of flexible microsystems including the incorporation of paper as a substrate ${ }^{18-21}$ and the use of inexpensive laser machining ${ }^{22-24}$. The use of paper simultaneously provides structural flexibility as well as selective filtering functionality, i.e., it allows for oxygen to pass through while preventing aqueous solutions to reach the 
tissue. The laser machining enables the precise definition of oxygen generating regions that match the hypoxic wound profile. The silicone-based composition of the paper's binder enables seamless integration with other flexible (e.g., PDMS) microfluidic and therapeutic components. This platform enables printing of oxygen generating materials as well as oxygen sensing flexible sensors. Together these two technologies allows the development of a low-cost wounddressing with customized, wound-specific oxygen generating regions which can be integrated with sensors and electronics to close the sensing and delivery loop for therapeutic adjustments. This manuscript focuses on the development of an oxygen generation platform using techniques that are scalable for large scale manufacturing.

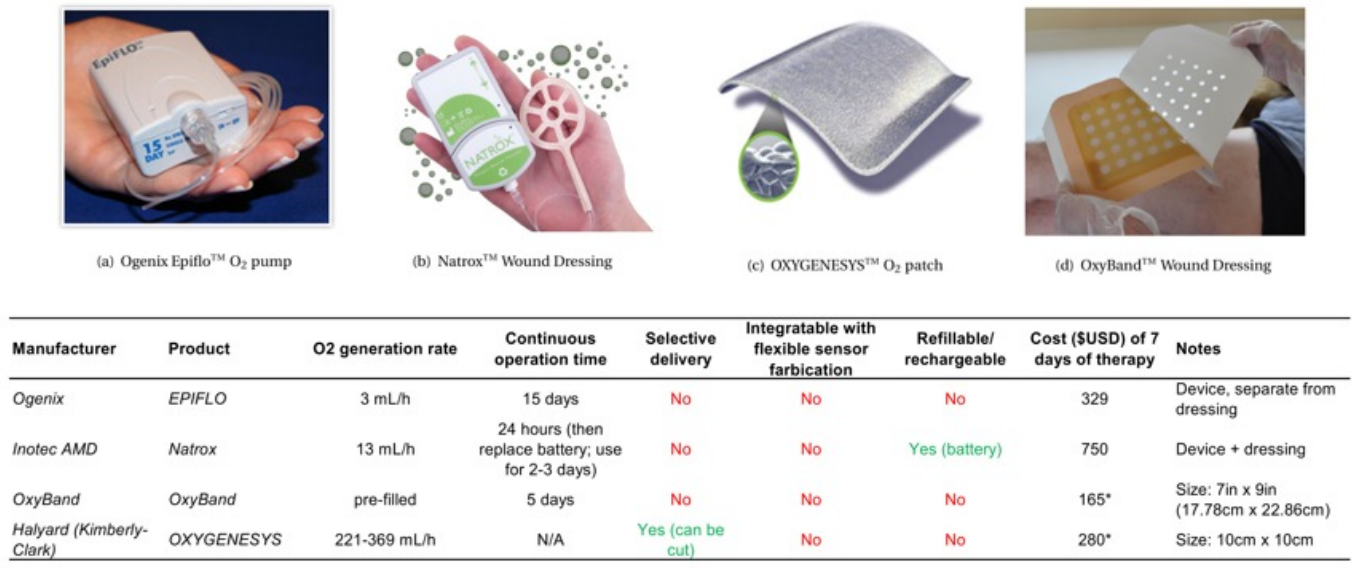

Figure 2: Comparison of several commercially available systems for topical $\mathrm{O}_{2}$ delivery; all supply $\mathrm{O}_{2}$ to the entire wound, without allowing selectivity towards hypoxic regions.

\section{DESIGN}

\subsection{Platform structure}

The oxygenation dressing consists of an inexpensive, paper-based, biocompatible, flexible platform for locally generating and delivering oxygen to selected hypoxic regions. It takes advantage of recent developments in the fabrication of flexible microsystems including the incorporation of paper as a substrate and the use of inexpensive laser machining and printing technologies. The platform is suitable for mass production, as it features layer-by-layer fabrication and components which can be printed via commercial inkjet/screen-printing. The platform consists of a flexible microfluidic network bonded to an active parchment paper substrate. A key feature is the use of laser-patterned parchment paper as the primary structural/functional material. Parchment paper is a hydrophobic material by design; however, it can be ablated using a $\mathrm{CO}_{2}$ laser to create hydrophilic regions ${ }^{25}$. This technique is applied to define an array of hydrophilic spots. The natural mesh structure of paper allows the spots to be embedded with chemicals suspended in an aqueous solution.

\subsection{Sensing and delivery mechanisms}

The paper substrate is patterned with a catalyst along the microfluidic network for the generation of oxygen. When $\mathrm{H}_{2} \mathrm{O}_{2}$ is injected through the microchannel network, it reaches the spot array and is decomposed by the catalyst, resulting in oxygen generation $^{26-28}$. The substrate can also be patterned concurrently with other active materials, such as colorimetric or fluorescent inks to enable optical sensing of oxygen alongside delivery. Figure 3 illustrates the principles of such generation and sensing. This paper focuses on the generation portion and highlights the suitability of patterned parchment paper for manufacturable wound dressing applications. 

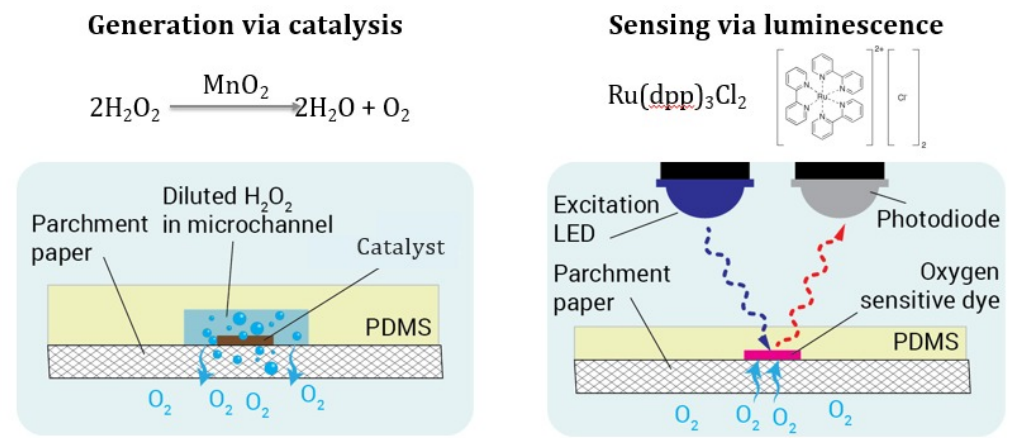

Figure 3: Mechanisms for generating (left) and sensing (right) oxygen for use on a flexible smart wound dressing.

A convenient way to oxygenate wounds is to generate oxygen within the wound dressing. An economical and broadly available option is the use of hydrogen peroxide, since it is readily available in the medical environment at safe-to-use concentrations $\left(3 \% ; 1 \mathrm{~mL}\right.$ of this produces $11.4 \mathrm{~mL}$ of $\mathrm{O}_{2}$ at $\left.37^{\circ} \mathrm{C}\right)$. Hydrogen peroxide is a commonly safe consumable that can be safely injected into aqueous systems without risk of producing unwanted contaminants. Hydrogen peroxide can be catalyzed by many transition metals and their compounds to produce oxygen via the reaction below.

$$
2 \mathrm{H}_{2} \mathrm{O}_{2} \rightarrow 2 \mathrm{H}_{2} \mathrm{O}+\mathrm{O}_{2}
$$

Of the various catalyst materials available, manganese dioxide stands out as a convenient option. Manganese dioxide micro-particles are biocompatible and catalyzes hydrogen peroxide while simultaneously possessing the additional advantages of being economical, simple to synthesize, and commercially available. In addition, the catalyst can be synthesized rapidly via a precipitation reaction (e.g., $\mathrm{KI}+\mathrm{KMnO}_{4}$ ) without requiring complex fabrication techniques. This process also allows facile deposition of the catalyst on various substrates via standard commercial manufacturing techniques (e.g., gravure, printing, inkjet printing, dip coating) by processing each of the two reactants sequentially. In the context of wound dressings, the ability to precipitate the catalyst in situ allows for deposition of the catalyst within the fibers of the wound dressing; in particular, the catalyst can be easily printed on laser-machined parchment paper, to create paper-based oxygenation wound dressings. Furthermore, it is possible to pre-treat the catalyst particles to achieve nano-scale surface features for enhanced catalytic activity while retaining its overall micro-scale (i.e., safe) size.

\section{EXPERIMENTAL}

\subsection{Characterization of laser ablated parchment paper as a substrate}

Characterization were performed on several common hydrophobic papers and films in terms of their change in surface properties after laser treatment, wet strength (standard tensile strain tests as a function of wetting duration), and nutrition/gas permeability. These films were parchment paper (PP), wax paper (WP), filer paper (FP), PDMS, and a paper-PDMS (P/PDMS) composite prepared by placing a sheet of filter paper on a thin layer $(50 \mu \mathrm{m})$ of PDMS prepolymer spin-coated onto a silanized silicon wafer and cured at $80^{\circ} \mathrm{C}$ for $30 \mathrm{~min}$. The surface properties of the films were characterized before and after laser irradiation $\left(\mathrm{CO}_{2}\right.$ pulsed laser operated at $10 \mathrm{~W}$ power and scanning speed of 35 $\mathrm{mm} / \mathrm{s})$. The optimized laser settings were sufficient to change the surface morphology and properties of the hydrophobic paper without completely cutting through the material. The surface wettability of parchment paper and other paper types was evaluated by measuring the static contact angle of a $10 \mu \mathrm{L}$ droplet of DI water before and after laser treatment using an optical contact angle measuring device (Rame-Hart goniometer, model 590). All experiments were conducted five times and the mean contact angle was calculated. High magnification surface and cross-sectional scanning electron microscopy (SEM) images were also obtained to assess the change in surface morphology on the laser ablated parchment paper. Standard tensile stress-strain tests were conducted to characterize the mechanical properties of the paper in dry and wet conditions. The wet paper samples were prepared by submerging them in buffer saline solution (PBS) for several durations (0-7 days), and the ultimate tensile strength (UTS) and Young's modulus were subsequently measured using a universal testing machine (Admet ${ }^{\circledR}$, model eXpert 1000). Oxygen permeability tests were performed in custom designed test setup that consisted of a cylindrical chamber filled with $20 \mathrm{~mL}$ of deoxygenated DI water covered by with a circular sample paper membrane. The dissolved oxygen was removed by purging the water with nitrogen gas for $8 \mathrm{~h}$ 
before each experiment. The oxygen permeability was confirmed with real-time the dissolved oxygen measurements using optical oxygen sensor (NeoFox, OceanOptics, Dunedin, FL) that was positioned in the DI water chamber.

\subsection{Preparation and characterizations of the catalyst}

$\mathrm{MnO}_{2}$ microparticles $\left(\sim 150 \mu \mathrm{m}\right.$ diameter) were obtained from Sigma-Aldrich and used as received. Smaller $\mathrm{MnO}_{2}$ particles $\left(\sim 20 \mu \mathrm{m}\right.$ diameter) were synthesized by reacting $5 \mathrm{~mL}$ of $0.1 \mathrm{~N} \mathrm{KMnO}_{4}$ with $5 \mathrm{~mL}$ of $0.1 \mathrm{~N}$ KI. Precipitates were collected by centrifugation and rinsed with deionized (DI) water three times to remove impurities. The particles were then dried in a nitrogen gas environment and maintained in an inert environment until just prior to usage.

The catalytic activity of the catalyst particles was investigated in bulk. For this, $20 \mathrm{mg}$ of the large-size catalyst powder was mixed in a flask with $10 \mathrm{~mL}$ of $30 \% \mathrm{H}_{2} \mathrm{O}_{2}$ and immediately connected to a standard trough setup for collection of generated gas. The volume of generated gas was measured over time by time-lapse photography; the generation rate of oxygen was subsequently calculated from the photographs. This experiment was repeated for the small-size particles (same mass of catalyst and peroxide concentration). The catalyst particle morphology was also investigated by imaging via scanning electron microscopy (SEM) before and after the catalytic activity experiments. The particle size of both catalyst samples was analyzed from the SEM images using optical granulometry software (ImageJ plugin).

\section{RESULTS AND DISCUSSION}

\subsection{Parchment paper characterizations}

Figure 4 shows the change in UTS and Young's modules for different papers/films before and after submersion in PBS. The dry samples exhibit a linear stress-strain profile with limited $(2.5 \%)$ elongation before rupture. The sharp drop in the stress indicates the strain at which the paper tears. The Young's modulus of the various papers is extracted from the stress-strain curves. The data reveal a high elastic modulus $(>100 \mathrm{kPa})$ for all dry samples, except for filter paper. Once wet, however, the elastic modulus of each sample drops below $50 \mathrm{kPa}$. However, the drop in the elastic modulus of parchment paper (from over $300 \mathrm{kPa}$ to about $45 \mathrm{kPa}$ ) is much smaller than the others. These values make parchment paper sufficiently rigid for handling during the fabrication and sufficiently soft when wet for wound dressing applications. Skin is known to have an elastic modulus of 24.910-101.180 kPa, depending on the skin location. Thus, wet parchment paper matches the elastic properties of the skin. As such, it can be compared to other commercial products, such as a commercial wound regeneration matrix (e.g., Integra Dermal Regeneration Matrix, Integra Life Sciences) which is used routinely in burn-related surgeries; this product has a Young's modulus of $25-45 \mathrm{kPa}$, in the lower range of that for parchmet paper.

Parchment paper is patterned with hydrophilic regions for subsequnt deposition of aquous inks for oxygen sensing and genration. When parchment paper is laser-machined, it results in a decreased water contact angle. Figure 5a,d show the contact angle of parchment paper changing from $121^{\circ}$ to $21^{\circ}$ as a result of laser treatment. This increase in surface wettability is due to the creation of exposed micro/nano cellulose fibers and addition of hydrophilic $-\mathrm{OH},=\mathrm{O}$ groups on the laser-ablated areas.

Laser treatment also causes change in morphology, as can be seen in Figure 5b, e. The SEM images shows the top view of the paper before and after laser treatment. The image show a clear change in morphology with exposed microlslash nano fibers on the surface of the paper after laser treatment. To more clearly observe the morphological changes, one can look at the cross-section of the parchment paper, Figures 5c,f. As evident in these images, the thickness of the paper changes as a result of laser treatment. While the initial thickness of the paper is $60 \mu \mathrm{m}$, the laser-ablated region was protruded out of the plane by $15 \mu \mathrm{m}$ over the original surface. This can be attributed to the decomposition/re-deposition of the silicone coating in the paper upon laser exposure, leading to the generation of higher-volume porous microlslash nano roughness. Together, the roughness and increased volume of the laser-machined parchment paper produce a sponge-like material into which microparticles can be loaded; this is useful for loading oxygen-generation catalysts, as is described in the following two chapters.

Figure 6 shows the permeability of dissolved oxygen in a wet environment (setup shown in inset). For all measurements the initial dissolved oxygen of the DI water was close to zero $(\sim 0.5 \mathrm{ppm})$ and increased with time up to the oxygen saturation level in the water $(8 \mathrm{ppm})$. The increase was due to the diffusion of the oxygen gas in ambient condition through the paper membrane and its dissolution in the water. Without any membrane, the water equilibrates to its steady- 
state saturation level of about $8 \mathrm{ppm}$ in less than $140 \mathrm{~min}$. However, when the chamber was covered, the time required for oxygen saturation increases. The results showed the longest oxygen equilibration time occurred for a pristine $100 \mu \mathrm{m}$ membrane of PDMS (720 min). Parchment paper had a larger oxygen permeability (time to saturation of 210 min, with an average rate of $2.4 \mathrm{ppm} / \mathrm{h}$ ). No signs of water leakage were observed with the hydrophobic films during any of the measurements. The mechanical strength and gas permeability results showed the superior performance of the parchment paper.

For wound dressing applications, patterning parchment paper allows the rapid creation of hydrophilic traces or regions onto which other materials can be deposited. In particular, parchment paper can be loaded with microparticles (e.g., chemical catalysts) for generating oxygen, as is described in the subsequent section. The paper is patterned as described above, and microparticles are subsequently deposited on the hydrophilic regions. Figure 7 displays the pattern definition capabilities of the process; the laser-ablated spots are clearly defined and their hydrophilicity allows for precise patterning of the catalyst. The spots have a diameter of $800 \mu \mathrm{m}$, but smaller (or larger) custom sizes are possible up to the resolution limit of the laser system $(125 \mu \mathrm{m}$ in our case). Magnified views of the catalyst is shown in the SEM images. The images show the increased uniformity and smaller particle size achievable with the reaction-deposition approach as opposed to the powder casting method. With the reaction approach, the wicking action of the paper in the catalyst spots absorbs each of the reactants, allowing the catalyst precipitate to be generated within the paper mesh for improved particle entrapment and reduced catalyst washout rate during operation.
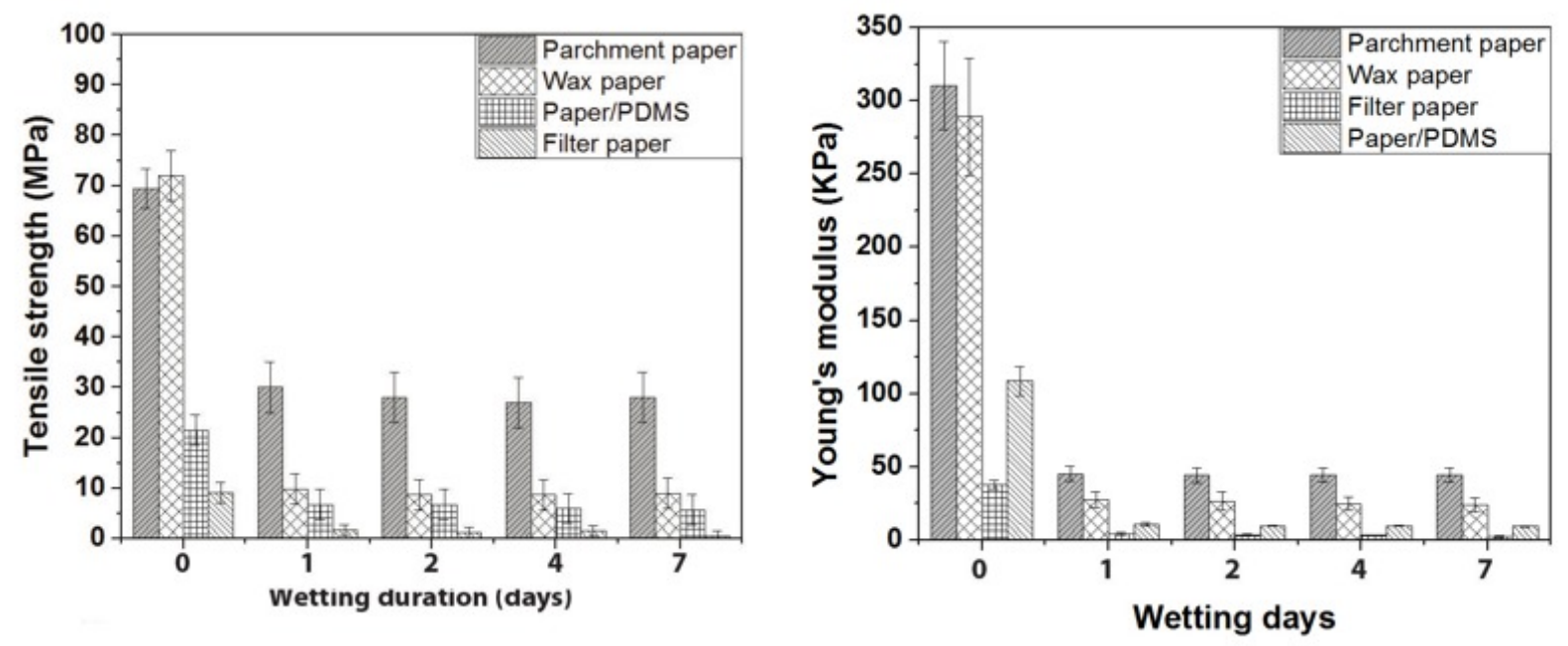

Figure 4: Ultimate tensile strength (UTS) and Young's modulus of various papers in dry and wet conditions. Reproduced ${ }^{31}$ with permission of the Royal Society of Chemistry. 


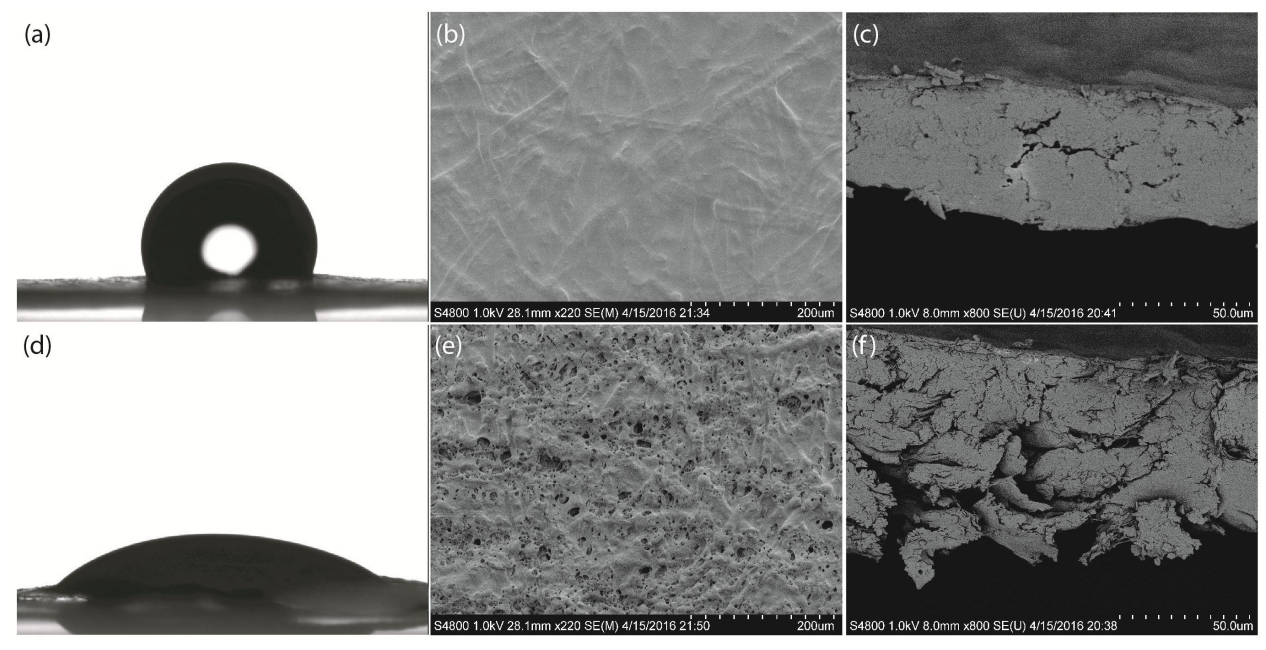

Figure 5: Surface properties of parchment paper before (a-c) and after (d-f) laser machining. Reproduced ${ }^{32}$ with permission from the Royal Society of Chemistry.
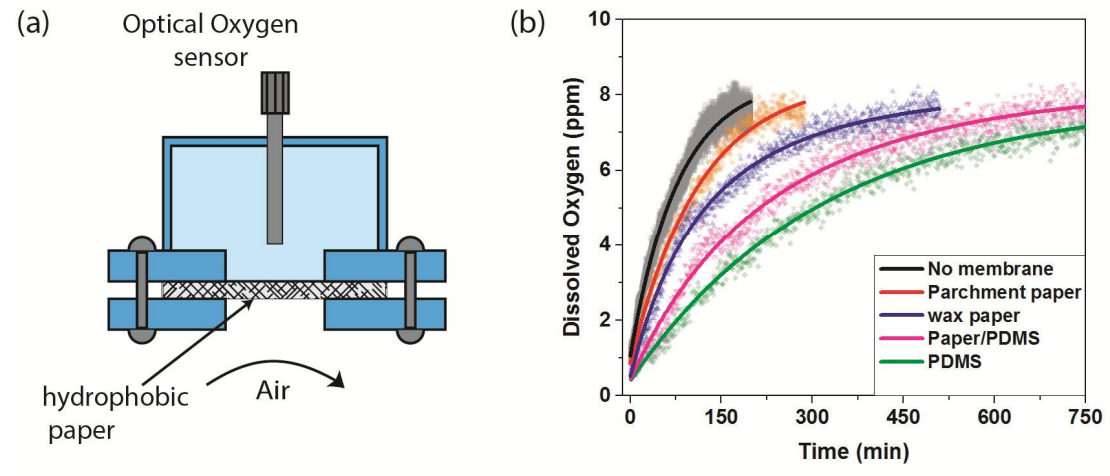

Figure 6: Oxygen diffusion of different hydrophobic papers. Reproduced ${ }^{31}$ with permission from The Royal Society of Chemistry.
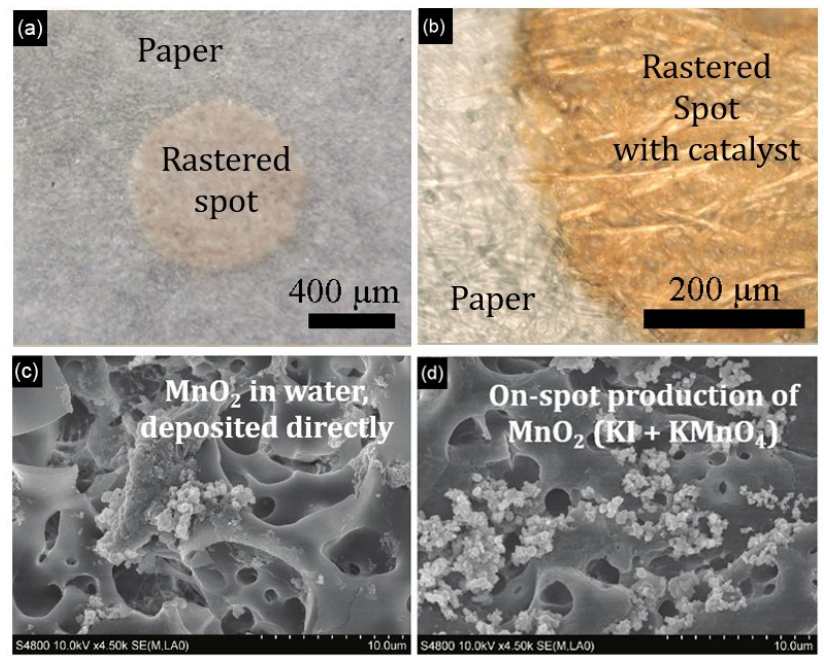

Figure 7: EM images of $\mathrm{MnO}_{2}$ particles in a laser-ablated spot on parchment paper. (a) Catalyst deposited as an aqueous suspension of $\mathrm{MnO}_{2}$ particles result in large material clumps on the paper surface. (b) Catalyst deposited by on-spot precipitation via the chemical reaction of two aqueous solutions of $\mathrm{KI}$ and $\mathrm{KMnO}_{4}$ results in smaller, more uniformly distributed and entrapped particles.

Reproduced $^{32}$ with permission from Elsevier. 


\subsection{Oxygen generation}

The characterization experiments for the catalyst reveal a drastic change in catalytic activity between the two particle sizes. The catalytic activity can be interpreted as the rate of oxygen generation in a given solution of $\mathrm{H}_{2} \mathrm{O}_{2}$. Figure 8 (a) shows the normalized gas generation $\left(\mu \mathrm{L} \mathrm{O}_{2}\right.$ per minute per $\mu \mathrm{g}$ of $\left.\mathrm{MnO}_{2}\right)$ for both particles during 20 minutes of immersion in the $30 \% \mathrm{H}_{2} \mathrm{O}_{2}$ solution. The data show up to an eight-fold increase in rate for the smaller ones, compared to the larger ones. Such large difference for the same mass is expected, since the smaller particles have a much higher surface area available for catalyzing the $\mathrm{H}_{2} \mathrm{O}_{2}$. Additionally, by the 6 minutes mark, the rate of oxygen generation for the small particles reaches at peak value which is 2.3 times its initial rate, whereas the rate of the large particles continues to increase monotonically, reaching nearly 6 times its original rate by the 20 minute mark, Figure 8(b). Thus, in this sense, the smaller particles offer a more stable rate of generation of oxygen, compared to the larger ones. These effects and catalytic rates provide a means for tuning the oxygenation activity during the design phase of wound dressings.

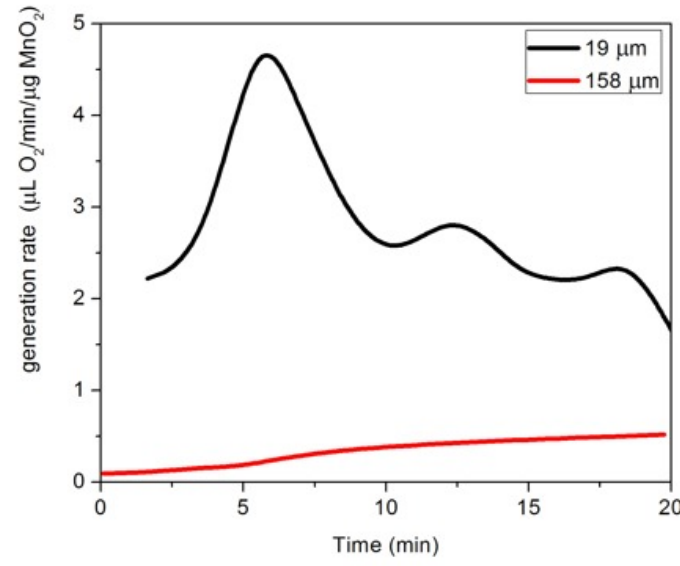

(a)

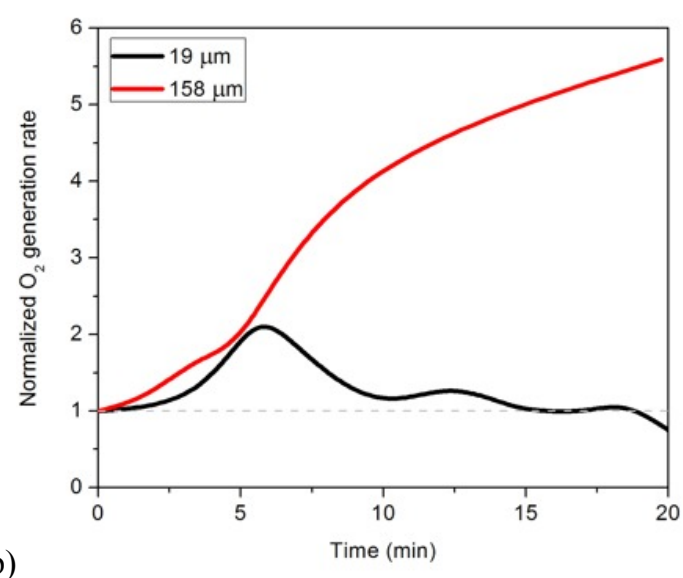

(b)

Figure 8: (a) Oxygen generation rate increases over time when immersed in $\mathrm{H} 2 \mathrm{O} 2$. The rate is up to 8 times higher for the smaller particles compared to the larger ones. (b) Normalized oxygen generation rate of the two particle sizes with respect to the initial rate of

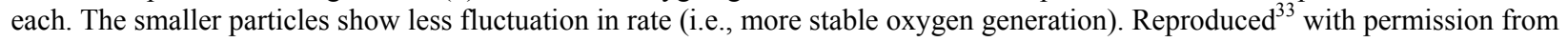

IEEE.

\section{CONCLUSIONS}

The overall performance of the oxygen generation platform is adequate for its intended application as a component of a disposable oxygen therapy wound dressings. Future development will focus on practical packaging measures necessary for clinical use. These include its incorporation into a commercial wound dressing as well as the implementation of an on-board hydrogen peroxide source. The microfluidic structure provides a convenient location for encapsulating $\mathrm{H}_{2} \mathrm{O}_{2}$ in a small $(1-10 \mathrm{~mL})$ pre-pressurized chamber that delivers a continuous flow through the microchannels. For example, a reservoir of dimensions $100 \mathrm{~mm} \times 100 \mathrm{~mm} \times 1 \mathrm{~mm}$ would contain enough $3 \% \mathrm{H}_{2} \mathrm{O}_{2}$ solution to generate about $100 \mathrm{~mL}$ $\mathrm{O}_{2}$, hence enabling a production rate of $100 \mathrm{~mL} \mathrm{O}_{2} /$ hour for up to $33 \mathrm{~h}$. The peroxide concentration and/or reservoir dimensions can be adjusted to optimize for platform size or oxygenation capacity. The oxygen release profile for completely packaged devices will be subsequently evaluated with in vitro and/or or in vivo experiments.

\section{REFERENCES}

[1] Medical Data International., "US market for chronic wound management products" (1997).

[2] Sen, C. K., Gordillo, G. M., Roy, S., Kirsner, R., Lambert, L., Hunt, T. K., Gottrup, F., Gurtner, G. C. and Longaker, M. T., "Human skin wounds: a major and snowballing threat to public health and the economy.," Wound Repair Regen. 17(6), 763-771 (2009).

[3] Stojadinovic, A., Carlson, J. W., Schultz, G. S., Davis, T. A. and Elster, E. A., "Topical advances in wound care.," Gynecol. Oncol. 111(2 Suppl), S70-80 (2008).

[4] Tarnuzzer, R. O. Y. W. and Schultz, G. S., "Biochemical analysis of acute and chronic wound environments," Wound Repair Regen. 4, 321-325 (1996). 
[5] Falanga, V., "Classifications for wound bed preparation and stimulation of chronic wounds," Wound Repair Regen. 8(5), 347-352 (2000).

[6] Castilla, D. M., Liu, Z.-J. and Velazquez, O. C., "Oxygen: Implications for Wound Healing,” Adv. Wound Care 1(6), 225-230 (2012).

[7] Greaves, N. S., Iqbal, S. a, Baguneid, M. and Bayat, A., "The role of skin substitutes in the management of chronic cutaneous wounds.," Wound Repair Regen. 21(2), 194-210 (2013).

[8] Lait, M. E. and Smith, L. N., "Wound management: a literature review," J. Clin. Nurs. 7(1), 11-17 (1998).

[9] Eaglstein, W. H., "Moist wound healing with occlusive dressings: a clinical focus.," Dermatol. Surg. 27(2), 175181 (2001).

[10] Chaby, G., Senet, P., Vaneau, M., Martel, P., Guillaume, J.-C., Meaume, S., Téot, L., Debure, C., Dompmartin, A., Bachelet, H., Carsin, H., Matz, V., Richard, J. L., Rochet, J. M., Sales-Aussias, N., Zagnoli, A., Denis, C., Guillot, B. and Chosidow, O., "Dressings for acute and chronic wounds: a systematic review.," Arch. Dermatol. 143(10), 1297-1304 (2007).

[11] Mehmood, N., Hariz, A., Fitridge, R. and Voelcker, N. H., "Applications of modern sensors and wireless technology in effective wound management.," J. Biomed. Mater. Res. B. Appl. Biomater. 102(4), 885-895 (2014).

[12] Ochoa, M., Rahimi, R. and Ziaie, B., "Flexible Sensors for Chronic Wound Management," IEEE Rev. Biomed. Eng. 7, 73-86 (2014).

[13] Sen, C. K., "Wound healing essentials: let there be oxygen.," Wound repair Regen. 17(1), 1-18 (2010).

[14] Schreml, S., Szeimies, R. M., Prantl, L., Karrer, S., Landthaler, M. and Babilas, P., "Oxygen in acute and chronic wound healing.," Br. J. Dermatol. 163(2), 257-268 (2010).

[15] Kranke, P., Bennett, M. H., Martyn-St James, M., Schnabel, A. and Debus, S. E., "Hyperbaric oxygen therapy for chronic wounds.," Cochrane database Syst. Rev. 4(4), CD004123 (2012).

[16] Howard, M. A., Asmis, R., Evans, K. K. and Mustoe, T. A., "Oxygen and wound care: A review of current therapeutic modalities and future direction," Wound Repair Regen. 21(4), 503-511 (2013).

[17] Gordillo, G. M., Schlanger, R., Wallace, W. a, Bergdall, V., Bartlett, R. and Sen, C. K., "Protocols for topical and systemic oxygen treatments in wound healing.," Methods Enzymol. 381(1968), 575-585 (2004).

[18] Russo, A., Ahn, B. Y., Adams, J. J., Duoss, E. B., Bernhard, J. T. and Lewis, J. A., "Pen-on-paper flexible electronics.," Adv. Mater. 23(30), 3426-3430 (2011).

[19] Cheng, C.-M., Mazzeo, A. D., Gong, J., Martinez, A. W., Phillips, S. T., Jain, N. and Whitesides, G. M., "Millimeter-scale contact printing of aqueous solutions using a stamp made out of paper and tape.," Lab Chip(1), 3201-3205 (2010).

[20] Martinez, A. W., Phillips, S. T., Butte, M. J. and Whitesides, G. M., "Patterned paper as a platform for inexpensive, low-volume, portable bioassays.," Angew. Chem. Int. Ed. Engl. 46(8), 1318-1320 (2007).

[21] Ding, Z., Wei, P., Chitnis, G. and Ziaie, B., "Ferrofluid-Impregnated Paper Actuators," J. Microelectromechanical Syst. 20(1), 59-64 (2011).

[22] Ochoa, M., Chitnis, G. and Ziaie, B., "Laser-micromachined cellulose acetate adhesive tape as a low-cost smart material,” J. Polym. Sci. Part B Polym. Phys. 51(17), 1263-1267 (2013).

[23] Klank, H., Kutter, J. P. J. P. and Geschke, O., "CO(2)-laser micromachining and back-end processing for rapid production of PMMA-based microfluidic systems.," Lab Chip 2(4), 242-246 (2002).

[24] Chitnis, G. and Ziaie, B., "Waterproof Active Paper via Laser Surface Micropatterning of Magnetic Nanoparticles.," ACS Appl. Mater. Interfaces 4(9), 4435-4439 (2012).

[25] Chitnis, G., Ding, Z., Chang, C.-L., Savran, C. A. and Ziaie, B., "Laser-treated hydrophobic paper: an inexpensive microfluidic platform.," Lab Chip 11(6), 1161-1165 (2011).

[26] Broughton, D. B. and Wentworth, R. L., "Mechanism of Decomposition of Hydrogen Peroxide Solutions with Manganese Dioxide. I," J. Am. Chem. Soc. 69(4), 741-744 (1947).

[27] Broughton, D. B., Wentworth, R. L. and Laing, M. E., "Mechanism of Decomposition of Hydrogen Peroxide Solutions with Manganese Dioxide. II," J. Am. Chem. Soc. 69(4), 744-747 (1947).

[28] Do, S.-H., Batchelor, B., Lee, H.-K. and Kong, S.-H., "Hydrogen peroxide decomposition on manganese oxide (pyrolusite): kinetics, intermediates, and mechanism.," Chemosphere 75(1), 8-12 (2009).

[29] Vol'nov, I. I., [Peroxides, Superoxides, and Ozonides of Alkali and Alkaline Earth Metals], Springer US, Boston, MA (1966).

[30] Burdon, R. H., "Superoxide and hydrogen peroxide in relation to mammalian cell proliferation," Free Radic. Biol. Med. 18(4), 775-794 (1995). 
[31] Rahimi, R., Htwe, S. S., Ochoa, M., Donaldson, A., Zieger, M., Sood, R., Tamayol, A., Khademhosseini, A., Ghaemmaghami, A. M. and Ziaie, B., "A paper-based: In vitro model for on-chip investigation of the human respiratory system," Lab Chip 16(22) (2016).

[32] Ochoa, M., Rahimi, R., Huang, T. L., Alemdar, N., Khademhosseini, A., Dokmeci, M. R. and Ziaie, B., "A paper-based oxygen generating platform with spatially defined catalytic regions," Sensors Actuators B Chem. 198, 472-478 (2014).

[33] Ochoa, M., Yoon, C. K. and Ziaie, B., "Laser-Fabricated, Self-Forming Swimmers With Catalytic Propulsion and Magnetic Navigation," J. Microelectromechanical Syst. 26(4) (2017).

[34] Özkar, S., "Enhancement of catalytic activity by increasing surface area in heterogeneous catalysis," Appl. Surf. Sci. 256(5), 1272-1277 (2009). 